\title{
Salivary Desulfovibrio Desulfuricans Level and Oral Hygiene Index for the Prediction of Colorectal Cancer in Chinese Patients
}

\section{Yao Wang}

Shanghai Institute of Digestive Disease

\section{Yao Zhang}

Shanghai Jiao Tong University School of Medicine Affiliated Renji Hospital

\section{Zheng Wang}

Shanghai Jiao Tong University School of Medicine Affiliated Renji Hospital

Jian Tang

Shanghai Jiao Tong University School of Medicine Affiliated Renji Hospital

\section{Dongxing Cao}

Shanghai Jiao Tong University School of Medicine Affiliated Renji Hospital

\section{Yun Qian}

Shanghai Institute of Digestive Disease

\section{Yuanhong Xie}

Shanghai Jiao Tong University School of Medicine Affiliated Renji Hospital

\section{Haiying Chen}

Shanghai Jiao Tong University School of Medicine Affiliated Renji Hospital

\section{Yingxuan Chen}

Shanghai Jiao Tong University School of Medicine Affiliated Renji Hospital Zhaofei Chen ( $\nabla$ zhaofei_chen@163.com)

Shanghai Jiao Tong University School of Medicine Affiliated Renji Hospital Jingyuan Fang ( $\nabla$ jingyuanfang@sjtu.edu.cn )

Shanghai Jiao Tong University School of Medicine Affiliated Renji Hospital

\section{Research}

Keywords: salivary microbiome, $\mathrm{CRC}$, oral hygiene index, Dd, nomogram

Posted Date: July 28th, 2020

DOI: https://doi.org/10.21203/rs.3.rs-44046/v1 
License: (c) (i) This work is licensed under a Creative Commons Attribution 4.0 International License. Read Full License 


\section{Abstract}

Background: Mounting evidence have shown that fecal microbiome can act as biomarkers for diagnosing colorectal cancer (CRC). Recent studies demonstrate that oral microbiome is concordant with gut microbiome. The role of oral microbiome in colorectal cancer has not been fully illustrated.

Methods: We collected preoperational saliva with a final cohort of 237 patients who underwent surgical resections or colorectal endoscopy in XX Hospital from January 2018 to January 2020. Clinical demographics, comorbidities and oral conditions were obtained from medical records or questionnaires. Salivary microbial biomarkers were detected by quantitative polymerase chain reaction (PCR) after DNA extraction. Multivariate logistics regression analysis was employed to analyze the risk factors for colorectal cancer. A four-variable prediction model was constructed based on the logistics analysis.

Results: Among the 237 patients enrolled, there were 95 endoscopy confirmed healthy control and 142 pathologically confirmed colorectal adenocarcinoma patients. Logistics regression analysis demonstrated that the risk factors associated with CRC included age at diagnosis (OR=1.111, $95 \% \mathrm{Cl}=1.072-1.151)$, male sex $(\mathrm{OR}=2.111,95 \% \mathrm{Cl}=1.068-4.175)$, oral hygiene index $(\mathrm{OR}=1.769$, $95 \% \mathrm{Cl}=1.116-2.804)$ and relative salivary Desulfovibrio desulfuricans $(\mathrm{Dd})$ abundance $(\mathrm{OR}=1.156$, $95 \% \mathrm{Cl}=1.05-1.272)$, based on which a four-variable model was developed. The four-variable model had good discriminative (Brier score $=0.144$, Concordance index $=0.866$ ) and calibration $(0.834)$ abilities after bias correction.

Conclusions: Elevated salivary Dd level is an independent risk factor for CRC. We have developed a fourvariable model that could help identify at-risk patients for CRC.

\section{Background}

Colorectal cancer (CRC) is one of the major public health problems with both high incidence and mortality rate $^{1}$. In China, annually there are approximately 376,300 newly diagnosed CRC cases and 191,000 CRC related deaths ${ }^{2}$. Early identification of $\mathrm{CRC}$ could significantly reduce cancer incidence and mortality rates $^{3}$. Classical CRC screening methods including serum carcinoembryonic antigen (CEA), colonoscopy and fecal occult bleeding test (FOBT) are widely used in clinical practice, the screening method, however, neither lack sensitivity nor is invasive and has low patient compliance ${ }^{4,5}$. Thus, a novel non-invasive biomarker with higher sensitivity and patient compliance is warranted.

Typically, colorectal tumorigenesis from precancerous adenoma to colorectal cancer involves several stepwise genetic events ${ }^{6}$. Numerous studies have reported that microbiota dysbiosis plays a pivotal role in the initiation and development of CRC and fecal microbiome act as biomarkers for diagnosing $\mathrm{CRC}^{7}$. Given the concordance and relevance of oral and gut microbiome ${ }^{8}$, many researchers started to explore the oral microbiome structure and function of $\mathrm{CRC}^{9}$. Nevertheless, these studies lack investigations on 
clinical parameters and oral health status, which might impact the composition of oral microbiota. In addition, qPCR technique-based detection of certain oral bacteria has not been reported.

Poor oral hygiene has been demonstrated to be associated with higher risk of oral cancer and head and neck cancer ${ }^{10,11}$. What's more, one research conducted in Austria reported that head and neck squamous cell carcinoma patients presented great needs of dental care, which was underestimated by most patients

12. Oral hygiene indicators comprise of tooth brushing frequency, number of loss teeth, regularity of dental visits and presence of dental caries.

Measuring salivary microbial biomarkers that have previously been identified for their possible role in carcinogenesis and assessing oral conditions, the current study aimed to establish a predictive model based on clinical characteristics, oral hygiene indicators and salivary microbiome for predicting the risk for colorectal cancer. We hypothesized that the combined use of oral hygiene index with biomarkers abundance could maximize the prediction accuracy of the model in diagnosing CRC.

\section{Methods}

\section{Patient Selection and Sample Collection}

Consecutive patients who underwent colonoscopy or surgical resections were enrolled in Shanghai Jiao Tong University affiliated Renji Hospital from Jan.2018 to Jan.2020. Patients with colonoscopy confirmed no neoplasms or pathologically diagnosed colorectal adenocarcinoma were assigned to healthy control $(\mathrm{HC})$ or CRC group, respectively. Strict exclusion criteria were applied to the subjects recruited as follows: 1) with history of gastrointestinal neoplasia; 2) with history of upper gastrointestinal tracts surgery; 3 ) specific types of CRCs including Lynch Syndrome, familial adenomatous polyposis (FAP) and Peutz-Jeghers syndromes; 4) after chemotherapy or radiotherapy; 5) medication history of any of the following drugs: NSAIDs (nonsteroidal anti-inflammatory drugs), immuno-inhibitors, antibiotics or probiotics for past one month before enrollment. The workflow chart was shown in Figure 1. A total number of 237 patients were included in the final statistical analysis. Preoperational unstimulated saliva was collected in a sterile container and transferred to $-80^{\circ} \mathrm{C}$ refrigerator in 30 minutes until future use. The procedures were all performed by specialized doctors who were blind to the research content. The current study has received the approval from Renji Hospital ethics committee and written informed consent was obtained from all subjects enrolled.

\section{Data Collection}

Patient demographics including age, sex, body weight, height, tobacco and alcohol use as well as comorbidities were either retrieved from the hospital medical system or obtained by face to face questionnaires. Body mass index (BMI) was calculated as weight $(\mathrm{kg})$ divided by height $(\mathrm{m})$ squared. Patients' self-reported oral hygiene information was obtained from questionnaires recorded by licensed doctors, which has been proven to be reliable ${ }^{13,14}$. Due to some restrictions to dental assessment, oral hygiene index $(\mathrm{OHI})$ was constructed based on three variables with minor modifications ${ }^{15}$. The three 
variables were scored categorically: for number of teeth loss $\geq 5$ scored 1 ; for tooth brushing frequency < twice $/$ day $=1$; irregular dental visits $=1$. Otherwise, the variable was scored 0 . The sum of the three variables equaled oral hygiene index, which ranged from 0 to 3 . As for the oral hygiene indicator for presence of dental caries, we defined it as a categorical variable. Tobacco or alcohol use was defined as consumption behavior for the past six months, no use or past consumption was defined as none-users. Comorbidities for diabetes or hypertension were also recorded as categorical variables.

\section{Genomic DNA Extraction and Quantitative PCR}

After thawing, saliva genomic DNA was extracted according to the manufacturer's instructions using QIAamp DNA mini kit (Qiagen, Germany). Upon DNA extraction, the purity and concentration of DNA were detected by Nanodrop 2000 spectrophotometer (Thermofisher Scientific, America) and stored at $-20^{\circ} \mathrm{C}$ until future use. Primers used for detecting 16S rRNA, Fn and Pg were extracted from previous $\operatorname{articles}^{16,17}$, while the primers for Dd and Pm were designed by Primer-BLAST in NCBI targeting the flavodoxin gene and hemolysin gene, respectively ${ }^{18,19}$. Primers used in this study were shown in supplementary materials. Quantitative PCR amplicons were performed in a $10 \mu$ reaction system of Sybergreen pre master mix containing in $A B I$ thermocycler (thermofisher). qPCR reactions were performed in duplicates for each target with the 16S rRNA as the internal control, which had been shown to indicate total bacteria DNA load ${ }^{20}$. qPCR conditions were as follows: $95^{\circ} \mathrm{C}$ denaturation for $5 \mathrm{~s}, 60^{\circ} \mathrm{C}$ annealing and extension for $30 \mathrm{~s}$ for a total of 40 cycles, after a short pre-denaturation at $95^{\circ} \mathrm{C}$ for $30 \mathrm{~s}$.

\section{Microbial Biomarker Selection}

In an attempt to search for salivary microbial biomarkers, the current study selected four pathogens as detection targets in the saliva, namely Fusobacterium nucleatum (Fn), Porphyromonas gingivalis $(\mathrm{Pg})$, Desulfovibrio desulfuricans (Dd) and Prevotella melaninogenica (Pm). Fn was among one of the most widely investigated pathogens associated with CRC, except for Fn in the body site of oral cavity. It was first identified that Fn was enriched in colorectal carcinoma tissues through whole genome sequencing, followed by quantitative PCR and $16 \mathrm{~S}$ rRNA sequencing analysis ${ }^{21}$. Fecal Fn has been widely reported as a diagnosis biomarker for $\mathrm{CRC}$ and high abundance of mucosa Fn was associated with chemotherapy resistance ${ }^{7,22}$. Mechanically, Fn could promote carcinogenesis through E-cadherin/ $\beta$-catenin signaling via FadA adhesin ${ }^{23}$. Pg was an Gram negative anaerobe associated with chronic periodontitis, which could eventually lead to tooth loss ${ }^{24}$. Pg acted as an important player in the development of various diseases, including Alzheimer's Disease ${ }^{25}$. The role of salivary Pg in colorectal cancer, however, remains unexplored. Dd is a Gram-negative anaerobe belonging to sulfate reducing bacteria (SRB). Functionally, SRB could degrade the organic matter entering into the gastrointestinal tract and utilize a wide range of substrates to reduce sulfate to hydrogen sulfide $(\mathrm{H} 2 \mathrm{~S})^{26}$. Bacteria derived $\mathrm{H} 2 \mathrm{~S}$ is toxic to colon epithelium, causing DNA damage to the colon epithelial cell and promoting colon cancer cell proliferation ${ }^{27,28}$. In vitro experiments showed that $\mathrm{Dd}$ endotoxin could transcriptionally activate NF-kB and IKB a genes in Caco2 colon cancer cell ${ }^{29}$. Recently, one research conducted in the United States 
revealed that sulfur metabolizing bacteria including Dd in the stool was associated with risk of distal colorectal cancer in $\mathrm{men}^{30}$. All of the above prompted us to investigate the role of salivary Dd in CRC. As for $\mathrm{Pm}$, prospective study demonstrated that $\mathrm{Pm}$ in pre-diagnostic mouth rinse samples was associated with a decreased risk of $\mathrm{CRC}^{31}$. Pm was enriched in tumoral microhabitat in a cohort of 276 gastric cancer patients ${ }^{32}$, suggesting a role of carcinogenesis in gastric cancer.

\section{Data Processing and Statistical Analysis}

For undetermined qPCR readouts, $\mathrm{Ct}$ values were replaced with a maximum value of 40 . The relative abundance of target salivary microbial biomarkers was denoted as target Ct value subtracted 16S rRNA Ct value. Kolmogorov-Smirnov normality test was first applied to assess the distribution of continuous data. Values were expressed as mean \pm standard deviation (SD) or median with interquartile range (IQR) or whole number with percentage as appropriate. Univariable comparisons of clinical characteristics and oral conditions between $\mathrm{CRC}$ and $\mathrm{HC}$ patients were made using independent $\mathrm{t}$ test (normal distribution) or Wilcoxon-Mann Whitney U nonparametric test (nonnormal distribution). For categorical variables, chisquare test was utilized. Factors independently associated with CRC were selected using multivariate logistics analysis with forward selection. Statistical analysis was all performed by SPSS version 25 (IBM). Missing data on salivary microbiome occurred because some patients left limited volume of saliva. Data on BMI (0.4\%), dental caries (2.5\%) and salivary microbiome level (2.5\% data for Fn, $2.1 \%$ for $\mathrm{Pg}, 0.4 \%$ for $\mathrm{Dd}$ and $2.1 \%$ for $\mathrm{Pm}$ ) were missing randomly and compared separately, but were multiply imputed as mean or median when analyzed in multivariate logistics analysis according to Rubin's rule ${ }^{33}$. A $P$ value less than 0.05 was considered as significant. Nomogram was constructed by R Package based on the odds ratio $(\mathrm{OR})$ calculated from multivariate logistics analysis.

\section{Results}

\section{Patient Characteristics and Oral Hygiene Indicators}

A total number of 237 patients met our criteria and were included in the final cohort, with 95 healthy control and 142 CRC patients (Fig. 1). The patients' overall characteristics, oral hygiene indicators and microbial biomarkers level were described as follows. Average age at diagnosis was $59.49 \pm 12.48$ years and sex was almost evenly distributed (men 53.16\%). The rate of comorbidity for hypertension in the cohort was $32.07 \%$, while for type 2 diabetes was $10.13 \%$. There was no significant difference between the two groups for patient $\mathrm{BMI}$ and drinking status $(P>0.05)$. However, there was significant difference for the variable age, sex, $\mathrm{OHI}$, the presence of dental caries, comorbidities and smoking status $(P<0.05)$. Average age at diagnosis was $51.16 \pm 10.75$ for $\mathrm{HC}$ group while CRC group was significantly older with average age at $65.07 \pm 10.27$. $\mathrm{HC}$ group had a lower male case rate $(44.21 \%$ vs $59.15 \%, P=0.024)$. OHI in CRC with a median value of 2 was higher than that of $\mathrm{HC}$ group with a median value of 1 , indicating worse oral hygiene habits for CRC group in comparison with $\mathrm{HC}$ group. A greater proportion of CRC patients had dental caries, comorbidities for type 2 diabetes and hypertension and smoking status. Univariate analysis between $\mathrm{HC}$ and CRC was summarized in Table 1. 
Table 1

Univariate Analysis of Characteristics and Oral Biomarkers of Recruited Patients between HC and CRC group.

\begin{tabular}{|llll|}
\hline Clinical and Oral Variables & HC $(\mathbf{n}=95)$ & CRC $(\mathbf{n}=142)$ & $P$ Value \\
\hline Age, mean (SD) & $51.16(10.75)$ & $65.07(10.27)$ & $<0.001$ \\
\hline Male, $n(\%)$ & $42,(44.21)$ & $84,(59.15)$ & 0.024 \\
\hline BMI, mean (SD) & $23.06(2.90)$ & $23.46(3.55)$ & 0.324 \\
\hline Dental caries, $n(\%)$ & $33(35.87)$ & $69(49.64)$ & 0.039 \\
\hline OHI median, (IQR) & $1(1-2)$ & $2(1-2)$ & $<0.001$ \\
\hline Comorbidities, $n(\%)$ & & $57,(40.14)$ & 0.001 \\
\hline Hypertension & $19,(20)$ & $21,(14.8)$ & 0.004 \\
\hline Type 2 diabetes & $3,(3.16)$ & $33,(23.24)$ & 0.041 \\
\hline Current smoking status, $\mathrm{n}(\%)$ & $12,(12.63)$ & $31,(21.83)$ & 0.96 \\
\hline Current alcohol use, $\mathrm{n}(\%)$ & $21,(22.11)$ & & 0.527 \\
\hline Biomarkers & & $9.91(8.799-11.216)$ & 0.001 \\
\hline Fn, median (IQR) & $10.125(9.1584-11.4306)$ & $<.1999(6.88-11.6946)$ & \\
\hline Pg, median (IQR) & $10.103(8.2519-13.7194)$ & 8.001 \\
\hline Dd, mean (SD) & $21.4573(3.28832)$ & $19.6226(3.64364)$ & 0.796 \\
\hline Pm, median (IQR) & $16.453(10.55-21.06)$ & $17.07(11.546-21.029)$ & \\
\hline Bold value signifies $P<0.05$ & & & \\
\hline SD indicates standard deviation, IQR indicates interquartile range. & \\
\hline
\end{tabular}

\section{Biomarker Comparison}

As for salivary microbial biomarkers, the relative abundance of salivary Fn and Pm showed no significant difference between the two groups. The relative abundance of salivary Pg and Dd in CRC is higher than that of the control group. Scatter plots of comparisons for the relative abundance of salivary $\mathrm{Pg}$ and $\mathrm{Dd}$ between $\mathrm{HC}$ and CRC group were shown in Fig. 2. The median relative $\mathrm{Pg} \mathrm{Ct}$ values were 10.11 and 6.88, while mean Dd Ct values were 21.47 and 19.63 for HC and CRC group, respectively. The optimal cut-off points for salivary $\mathrm{Pg}$ or $\mathrm{Dd}$ alone for discriminating $\mathrm{CRC}$ from $\mathrm{HC}$ were 8.31 and 21.95 , respectively. At these two cut-off points, area under receiver operation curve were 0.629 and 0.653 respectively. In all the 237 subjects enrolled, we identified a positive correlation of $\mathrm{OHI}$ with salivary $\mathrm{Pg}$ or Dd relative abundance (Spearman correlation coefficient $=0.188$ and $0.133, P=0.004$ and 0.04 , respectively). 


\section{Model Development And Nomogram Creation}

Missing values were imputed as appropriate before subjected to multivariate logistics regression analysis with forward selection. As demonstrated in Table 2, logistics regression analysis revealed that factors associated with $\mathrm{CRC}$ included age $(\mathrm{OR}=1.111,95 \% \mathrm{Cl}=1.072-1.151)$, male sex $(\mathrm{OR}=2.111,95 \% \mathrm{Cl}=$ 1.068-4.175), $\mathrm{OHI}(\mathrm{OR}=1.769,95 \% \mathrm{Cl}=1.116-2.804)$ and relative salivary $\mathrm{Dd}$ abundance $(\mathrm{OR}=1.156$, $95 \% \mathrm{Cl}=1.05-1.272)$. A four-variable model was constructed based on the four risk factors derived from logistics analysis. All variables included in the model showed no collinearity between each other (Variance Inflation Factor, VIF ranged from 1 to 2). Moreover, we developed a nomogram based on the ORs calculated from logistics regression (Fig. 3). Nomograms graphically present a complex mathematical algorithm, which incorporate biological and clinical variables ${ }^{34}$. The uppermost line was set as the reference for scoring points from 0 to 100, predictive variables were displayed right below with regularly segmented bars demonstrating visually the relative weight of each variable and allowing for values to be assigned to each variable accordingly. Total points for the nomogram and the corresponding predicted probability of CRC are shown in the bottom two bars.

Table 2

Multivariate Logistics Analysis for identifying independent risk factors for CRC

\begin{tabular}{|llll|}
\hline Variable & OR & $95 \% \mathrm{Cl}$ & $\boldsymbol{P}$ \\
\hline Age & 1.111 & $1.072-1.151$ & $<\mathbf{0 . 0 0 1}$ \\
\hline Sex & 2.111 & $1.067-4.174$ & $\mathbf{0 . 0 3 2}$ \\
\hline OHI & 1.769 & $1.116-2.804$ & $\mathbf{0 . 0 1 5}$ \\
\hline Salivary Dd & 1.156 & $1.05-1.272$ & $\mathbf{0 . 0 0 3}$ \\
\hline \multicolumn{2}{|l}{ Bold value signifies $P$ value $<0.05$} \\
\hline
\end{tabular}

\section{Evaluation of the Model}

The predictive accuracy of the four-variable model was evaluated using Brier score, Hosmer-Lemeshow goodness of fit test, calibration curve and concordance-index (C-index). Brier score was used to assess the difference between observed and predicted values with values closer to 0 indicating better predictive ability. By contrast, Hosmer-Lemeshow goodness of fit test is an over-all assessment of the difference between the predicted values and actual values with a $P$ value over than 0.05 indicating no difference and good calibration ability. As for calibration slope, it reflected the agreement between observed and predicted values with values closer to 1 indicating better performance. C-index was used for assessing the discriminatory ability of the nomogram with values between 0.5 and $1^{35}$. Bootstrapping with 237 repetitions was used for internal validation, and the bias-corrected accuracy measures of our predictive model were obtained. Brier score was 0.144 , which indicated good predictive ability of the model. Hosmer-Lemeshow goodness of fit test $P$ value equaled 0.054 , indicating no statistical difference 
between the predictive model and actual values. Calibration slope and C-index was rather high, with a value of 0.834 and 0.866 respectively. In addition, calibration curve and receiver operating characteristic curve were also displayed (Fig. 4) to graphically demonstrate calibration and discrimination abilities of the model, respectively.

\section{Discussion}

The oral cavity inhabits various microorganisms, whose diversity is only secondary to gut microbiome ${ }^{35}$. Oral microbiome has been associated with human health and diseases, including oral cancer ${ }^{37}$, pancreatic cancer ${ }^{38}$ and systemic diseases ${ }^{39}$. One prospective study of oral microbiome and colorectal cancer risk was conducted in African American populations ${ }^{31}$, nevertheless the oral microbiome was represented by mouth rinse samples and low-income populations were recruited so selection bias was possible. Saliva is fluid secreted by salivary glands with biological functions ${ }^{40}$, where salivary microbiome diversity exhibits temporal stability ${ }^{41}$, making it as optimal research target. In a study conducted by Italian scholars, preliminary comparisons of oral and gut microbiota in colorectal cancer patients were made and a different taxonomic composition was identified in CRC patients compared with HC group ${ }^{9}$. However, it neither lack strict inclusion and exclusion criteria nor the sample size was limited. In addition, 16S rRNA high throughput sequencing but not quantitative PCR based oral microbial biomarker detection was employed, which was cost consuming. In the present study, we utilized quantitative PCR to detect the relative abundance of specific pathogens in the saliva.

As for searching candidate salivary biomarkers, Fn, Pg, Dd and Pm were selected as qPCR targets in our cohort based on previous literatures. One recent study reported that salivary Fn level in CRC group was higher compared with the control group, while the amount of salivary Pg was similar between the two groups $^{42}$. However, the sample size included was limited which need further validation. Oral carriage of $\mathrm{Pg}$ has been demonstrated to be associated with increased risk of pancreatic cancer in a prospective study $^{38}$. The presence of SRB including Dd in the periodontal pocket and co-occurrence with Pg was observed, the prevalence of which was reduced after treatment ${ }^{43}$. As a matter of fact, we also found a positive correlation between salivary $\mathrm{Pg}$ and $\mathrm{Dd}$ level in our cohort (spearman correlation coefficient = $0.269, P<0.001)$. As for salivary $P m$, it has been reported to be a diagnostic indicator of oral squamous cell carcinoma ${ }^{44}$. The diagnostic role of salivary Pm in CRC still remains unexplored.

In our study, both salivary Pg and Dd were associated with the diagnosis of CRC in univariate analysis ( $P$ $<0.01)$. After adjusting for other risk factors, only Dd become the independent risk factor for CRC (OR = $1.156,95 \% \mathrm{Cl}=1.05-1.272)$. However, the discriminative ability of this salivary microbial biomarker in differentiating CRC from $\mathrm{HC}$ was limited when solely used (AUC $=0.653$, sensitivity $46.32 \%$, specificity 82.39\%). In addition, three other risk factors were also included in the logistics regression analysis, namely older age, male sex and higher oral hygiene index. As mentioned before, though we did have observed a positive correlation between $\mathrm{OHI}$ and relative salivary $\mathrm{Dd}$ abundance, the collinearity between the two predictive variables included in the model disappeared after adjustment for other risk factors for 
CRC $(1<\mathrm{VIF}<2)$. According to latest statistics ${ }^{1}$, the incidence rates for male and older age are higher, especially for age over than 50 , which is concordant with our results. High oral hygiene index was inversely correlated with good oral hygiene habits, which was demonstrated as a risk factor for $\mathrm{CRC}$. One retrospective research from Nurses' Health Study found that women with fewer teeth and moderate to severe periodontal diseases might be associated with increased risk of developing $\mathrm{CRC}^{45}$, which is consistent with ours. We expectedly observed a higher incidence rate for type 2 diabetes comorbidity in CRC, concordant with previous findings that type 2 diabetes mellitus was associated with higher risk of CRC from a prospective cohort ${ }^{46}$. Nevertheless, due to the low proportion of diabetes comorbidity rate in our cohort, this variable does not enter into final prediction model.

Colonoscopy is currently the most widely used screening method for detecting colorectal neoplasia, however, it's rather invasive, expensive and has low patient compliance 4 . Less invasive screening methods include FOBT and serum CEA and CA199 (carbohydrate antigen199) detection. Although FOBT has been demonstrated to reduce mortality rates, its sensitivity is limited ${ }^{5}$. Serum CEA is a relatively mature biomarker used in clinical practice to monitor CRC recurrence and response to therapy, but lacks sensitivity and specificity when used as a screening method ${ }^{47}$. Elevated serum CA199 is usually seen in late stage CRC and demonstrated good value in monitoring the prognosis of CRC ${ }^{48}$. Hence, there's an urgent need to develop a novel classifier for predicting the risk for CRC with less invasiveness and more convenience and patient compliance.

Nomograms have long been used by oncologists to predict cancer survival and metastasis ${ }^{49,50}$. Based on the coefficients calculated from multivariate logistics analysis, we assigned a value to each risk factor to calculate the total score capable of quantifying the nomogram. In the current study we developed a visually nomogram to predict the risk for CRC. In addition, we finally developed a four-variable model to predict CRC and internally validated its prediction accuracy. Derived from logistics analysis, our fourvariable model had a significantly higher AUC value compared with solo Dd used $(0.866$ vs $0.653, P<$ 0.0001 ), with a significantly higher sensitivity of $85.26 \%$ and a comparable specificity of $80.99 \%$. In addition, the predictive accuracy of the model was internally validated by bootstrap resampling as assessed by C-index, Brier score, Hosmer-Lemeshow test and calibration slope. Hosmer-Lemeshow test $(P=0.054)$ showed that the predicted value by the model had no significant difference with the actual value. The value of the $\mathrm{C}$-index for our model is 0.866 , which indicated a rather high concordance. Calibration slope of the model was performed by comparing the predicted value with the observed value after bias correction, which demonstrated a value of 0.834 . The Brier score is a parameter of evaluating the overall performance of the model, which equals 0.144 .

To our knowledge, this is the first oral microbiome based predictive model incorporating oral hygiene habits and salivary Dd level developed for diagnosing CRC. Our research expands the scope of CRC risk factors, such as poor oral hygiene habits and elevated salivary Dd level. Other than the aforementioned two variables, the remaining two variables included in the model are well established risk factors for CRC incidence. Most importantly, the four variables selected in the model were easy to collect with utmost 
convenience. What's more, the performance of the predictive model is encouraging as it demonstrated a high predictive ability for CRC, with both high sensitivity and specificity. Given the fact that continuous variable can be transformed to a score, constructing a nomogram is superior than other statistical models.

However, our research also has some limitations. This is a single-centered study which may not be generalizable. Our predictive model needs further external validation in the future. In addition, there is inevitable selection bias for the variable age during patient enrollment process, because there is significant difference for the age between outpatients for medical examinations and inpatients prepared for surgery.

\section{Conclusions}

our results demonstrate that elevated salivary Dd level is a risk factor for CRC, even after adjusting for other risk factors and salivary microbial biomarkers. We have developed a highly predictive model that can accurately differentiate CRC patients from those without colorectal neoplasm with great clinical applications. Given the fact that high oral hygiene index is a risk factor for CRC, developing good oral hygiene habits may help reduce the risk of CRC. However, the four-variable model should be externally validated in other centers and prospective researches should be conducted to evaluate the risk predicting value of aforementioned predictive variables before further clinical application. Additional researches are warranted to explore a mechanistic role for Dd in the initiation and development of CRC.

\section{Abbreviations}

$\mathrm{CRC}$, colorectal cancer; $\mathrm{PCR}$, polymerase chain reaction; $\mathrm{OR}$, odds ratio; $\mathrm{Cl}$ confidence interval; CEA, carcinoembryonic antigen; FOBT, fecal occult bleeding test;NSAIDs (nonsteroidal anti-inflammatory drugs); $\mathrm{HC}$, healthy control; BMI:body mass index; $\mathrm{OHI}$ :oral hygiene index; Fn, Fusobacterium nucleatum; Pg, Porphyromonas gingivalis; Dd, Desulfovibrio desulfuricans; Pm, Prevotella melaninogenica; SD, standard deviation; IQR, interquartile range; SRB, sulfate reducing bacteria; VIF, variance inflation factor; AUC, area under curve; CA199, carbohydrate antigen199.

\section{Declarations}

\section{Ethics approval and consent to participate:}

The current study has received the approval from Renji Hospital ethics committee and written informed consent was obtained from all subjects enrolled.

\section{Consent for publication:}

Not applicable. 
Availability of data and materials:

Not applicable.

\section{Competing interests:}

The authors declare no potential conflicts of interest.

\section{Funding:}

This study was funded by National Natural Science Foundation of China Scientific Research and Innovation Group (81421001) and National Natural Science Foundation of China (81772517).

\section{Author contributions:}

Conceptualization: YW, Z-FC, J-YF; Investigation: Z-FC, YZ, ZW, JT, D-XC, H-YC, Y-XC; Questionnaires: YW, Z-FC, YQ; Statistical Analysis: YW, YHX, J-YF; Manuscript Construction: YW, Z-FC, J-YF; Supervision and Funding: Z-FC, J-YF

\section{Acknowledgements:}

We thank Dr. Lu for dealing with R software.

\section{References}

1. Siegel RL, Miller KD, Jemal A. Cancer statistics, 2020. CA Cancer J Clin. 2020;70(1):7-30.

2. Chen W, Zheng R, Baade PD, et al. Cancer statistics in China, 2015. CA Cancer J Clin. 2016;66(2):115132.

3. Schoen RE, Pinsky PF, Weissfeld JL, et al. Colorectal-cancer incidence and mortality with screening flexible sigmoidoscopy. N Engl J Med. 2012;366(25):2345-2357.

4. Lieberman D A. Screening for Colorectal Cancer[J]. The New England Journal of Medicine, 2009: 9.

5. Hardcastle JD, Chamberlain JO, Robinson MHE, et al. Randomised controlled trial of faecal-occultblood screening for colorectal cancer. THE LANCET. 1996;348:6.

6. Fearon ER, Vogelstein B. A genetic model for colorectal tumorigenesis. Cell. 1990;61(5):759-767.

7. Liang Q, Chiu J, Chen Y, et al. Fecal Bacteria Act as Novel Biomarkers for Noninvasive Diagnosis of Colorectal Cancer. Clin Cancer Res. 2017;23(8):2061-2070.

8. Zhang $\mathrm{X}$, Zhang $\mathrm{D}$, Jia $\mathrm{H}$, et al. The oral and gut microbiomes are perturbed in rheumatoid arthritis and partly normalized after treatment. Nat Med. 2015;21(8):895-905. 
9. Russo E, Bacci G, Chiellini C, et al. Preliminary Comparison of Oral and Intestinal Human Microbiota in Patients with Colorectal Cancer: A Pilot Study. Front Microbiol. 2018;8:2699.

10. Gupta B, Bray F, Kumar N, Johnson NW. Associations between oral hygiene habits, diet, tobacco and alcohol and risk of oral cancer: A case-control study from India. Cancer Epidemiol. 2017;51:7-14.

11. Hashim D, Sartori S, Brennan $P$, et al. The role of oral hygiene in head and neck cancer: results from International Head and Neck Cancer Epidemiology (INHANCE) consortium. Ann Oncol Off J Eur Soc Med Oncol. 2016;27(8):1619-1625.

12. Bertl K, Loidl S, Kotowski U, et al. Oral health status and dental care behaviours of head and neck cancer patients: a cross-sectional study in an Austrian tertiary hospital. Clin Oral Investig. 2016;20(6):1317-1327.

13. Buhlin K, Gustafsson A, Andersson K, Håkansson J, Klinge B. Validity and limitations of self-reported periodontal health. Community Dent Oral Epidemiol. 2002;30(6):431-437.

14. Gilbert GH, Duncan RP, Kulley AM. Validity of self-reported tooth counts during a telephone screening interview. J Public Health Dent. 1997;57(3):176-180.

15. Ahrens W, Pohlabeln H, Foraita R, et al. Oral health, dental care and mouthwash associated with upper aerodigestive tract cancer risk in Europe: The ARCAGE study. Oral Oncol. 2014;50(6):616-625.

16. Xie Y-H, Gao Q-Y, Cai G-X, et al. Fecal Clostridium symbiosum for Noninvasive Detection of Early and Advanced Colorectal Cancer: Test and Validation Studies. EBioMedicine. 2017;25:32-40.

17. Dominy SS, Lynch C, Ermini F, et al. Porphyromonas gingivalis in Alzheimer's disease brains: Evidence for disease causation and treatment with small-molecule inhibitors. Sci Adv. 2019;5(1):eaau3333.

18. Helms LR, Swenson RP. Cloning and characterization of the flavodoxin gene from Desulfovibrio desulfuricans. Biochim Biophys Acta BBA - Gene Struct Expr. 1991;1089(3):417-419. doi:10.1016/0167-4781(91)90190-W

19. Allison HE, Hillman JD. Cloning and characterization of a Prevotella melaninogenica hemolysin. Infect Immun. 1997;65(7):2765-2771.

20. Kostic $A D$, Chun $E$, Robertson $L$, et al. Fusobacterium nucleatum potentiates intestinal tumorigenesis and modulates the tumor-immune microenvironment. Cell Host Microbe. 2013;14(2):207-215.

21. Kostic AD, Gevers D, Pedamallu CS, et al. Genomic analysis identifies association of Fusobacterium with colorectal carcinoma. Genome Res. 2012;22(2):292-298.

22. Yu T, Guo F, Yu Y, et al. Fusobacterium nucleatum Promotes Chemoresistance to Colorectal Cancer by Modulating Autophagy. Cell. 2017;170(3):548-563.e16.

23. Rubinstein MR, Wang X, Liu W, Hao Y, Cai G, Han YW. Fusobacterium nucleatum promotes colorectal carcinogenesis by modulating E-cadherin/ $\beta$-catenin signaling via its FadA adhesin. 2014:20.

24. Bostanci N, Belibasakis GN. Porphyromonas gingivalis: an invasive and evasive opportunistic oral pathogen. FEMS Microbiol Lett. 2012;333(1):1-9. 
25. Taylor JJ, Preshaw PM, Lalla E. A review of the evidence for pathogenic mechanisms that may link periodontitis and diabetes. J Clin Periodontol. 2013;40:S113-S134.

26. Scanlan PD, Shanahan F, Marchesi JR. Culture-independent analysis of desulfovibrios in the human distal colon of healthy, colorectal cancer and polypectomized individuals: Desulfovibrio sp. in the human distal gut. FEMS Microbiol Ecol. 2009;69(2):213-221.

27. Guo F-F, Yu T-C, Hong J, Fang J-Y. Emerging Roles of Hydrogen Sulfide in Inflammatory and Neoplastic Colonic Diseases. Front Physiol. 2016;7.

28. Linden DR. Hydrogen Sulfide Signaling in the Gastrointestinal Tract. Antioxid Redox Signal. 2014;20(5):818-830.

29. KAPRALa M. Quantitative Evaluation of Transcriptional Activation of NF- $\mathrm{B}$ p 65 and p50 Subunits and IIB Encoding Genes in Colon Cancer Cells by Desulfovibrio desulfuricans Endotoxin. 2010;55:5.

30. Nguyen LH, Ma W, Wang DD, et al. Association Between Sulfur-Metabolizing Bacterial Communities in Stool and Risk of Distal Colorectal Cancer in Men. Gastroenterology. 2020;158(5):1313-1325.

31. Yang Y, Cai Q, Shu X-O, et al. Prospective study of oral microbiome and colorectal cancer risk in lowincome and African American populations. Int J Cancer. 2019;144(10):2381-2389.

32. Liu X, Shao L, Liu X, et al. Alterations of gastric mucosal microbiota across different stomach microhabitats in a cohort of 276 patients with gastric cancer. EBioMedicine. 2019;40:336-348.

33. Rubin DB, Schenker N. Multiple imputation in health-care databases: an overview and some applications. Stat Med. 1991;10(4):585-598.

34. Grimes DA. The nomogram epidemic: resurgence of a medical relic. Ann Intern Med. 2008;149(4):273-275.

35. Harrell FE. Regression modeling strategies, with applications to linear models, survival analysis and logistic regression. GET ADDRESS Springer. 2001.

36. Kilian M, Chapple ILC, Hannig M, et al. The oral microbiome - an update for oral healthcare professionals. Br Dent J. 2016;221(10):657-666.

37. Gholizadeh P, Eslami H, Yousefi M, Asgharzadeh M, Aghazadeh M, Kafil HS. Role of oral microbiome on oral cancers, a review. Biomed Pharmacother. 2016;84:552-558.

38. Fan X, Alekseyenko AV, Wu J, et al. Human oral microbiome and prospective risk for pancreatic cancer: a population-based nested case-control study. Gut. 2018;67(1):120-127.

39. Kumar PS. Oral microbiota and systemic disease. Anaerobe. 2013;24:90-93.

40. Soini HA, Klouckova I, Wiesler D, et al. Analysis of Volatile Organic Compounds in Human Saliva by a Static Sorptive Extraction Method and Gas Chromatography-Mass Spectrometry. J Chem Ecol. 2010;36(9):1035-1042.

41. Cameron SJS, Huws SA, Hegarty MJ, Smith DPM, Mur LAJ. The human salivary microbiome exhibits temporal stability in bacterial diversity. Marchesi J, ed. FEMS Microbiol Ecol. 2015;91(9):fiv091.

42. Guven DC, Dizdar O, Alp A, et al. Analysis of Fusobacterium nucleatum and Streptococcus gallolyticus in saliva of colorectal cancer patients. Biomark Med. 2019;13(9):725-735. 
43. Langendijk-Genevaux PS, Grimm WD, van der Hoeven JS. Sulfate-reducing bacteria in relation with other potential periodontal pathogens. J Clin Periodontol. 2001;28(12):1151-1157.

44. Mager DL, Haffajee AD, Devlin PM, Norris CM, Posner MR, Goodson JM. The salivary microbiota as a diagnostic indicator of oral cancer: a descriptive, non-randomized study of cancer-free and oral squamous cell carcinoma subjects. J Trans/ Med. 2005;3:27-27.

45. Momen-Heravi F, Babic A, Tworoger SS, et al. Periodontal disease, tooth loss and colorectal cancer risk: Results from the Nurses' Health Study. Int J Cancer. 2017;140(3):646-652.

46. Ma Y, Yang W, Song M, et al. Type 2 diabetes and risk of colorectal cancer in two large U.S. prospective cohorts. Br J Cancer. 2018;119(11):1436-1442. doi:10.1038/s41416-018-0314-4

47. Fakih MG, Padmanabhan A. CEA monitoring in colorectal cancer. What you should know. Oncol Williston Park N. 2006;20(6):579.

48. Wang R, Song B-R, Peng J-J, et al. The Prognostic Value of Preoperative Serum CEA and CA19-9 Values in Stage I-III Colorectal Cancer. Hepatogastroenterology. 2014;61(132):994-999.

49. Iasonos A, Schrag D, Raj GV, Panageas KS. How to build and interpret a nomogram for cancer prognosis. J Clin Oncol Off J Am Soc Clin Oncol. 2008;26(8):1364-1370.

50. Huang $\mathrm{Y}-\mathrm{Q}$, Liang $\mathrm{C}-\mathrm{H}, \mathrm{He} \mathrm{L}$, et al. Development and Validation of a Radiomics Nomogram for Preoperative Prediction of Lymph Node Metastasis in Colorectal Cancer. J Clin Oncol Off J Am Soc Clin Oncol. 2016;34(18):2157-2164.

\section{Figures}




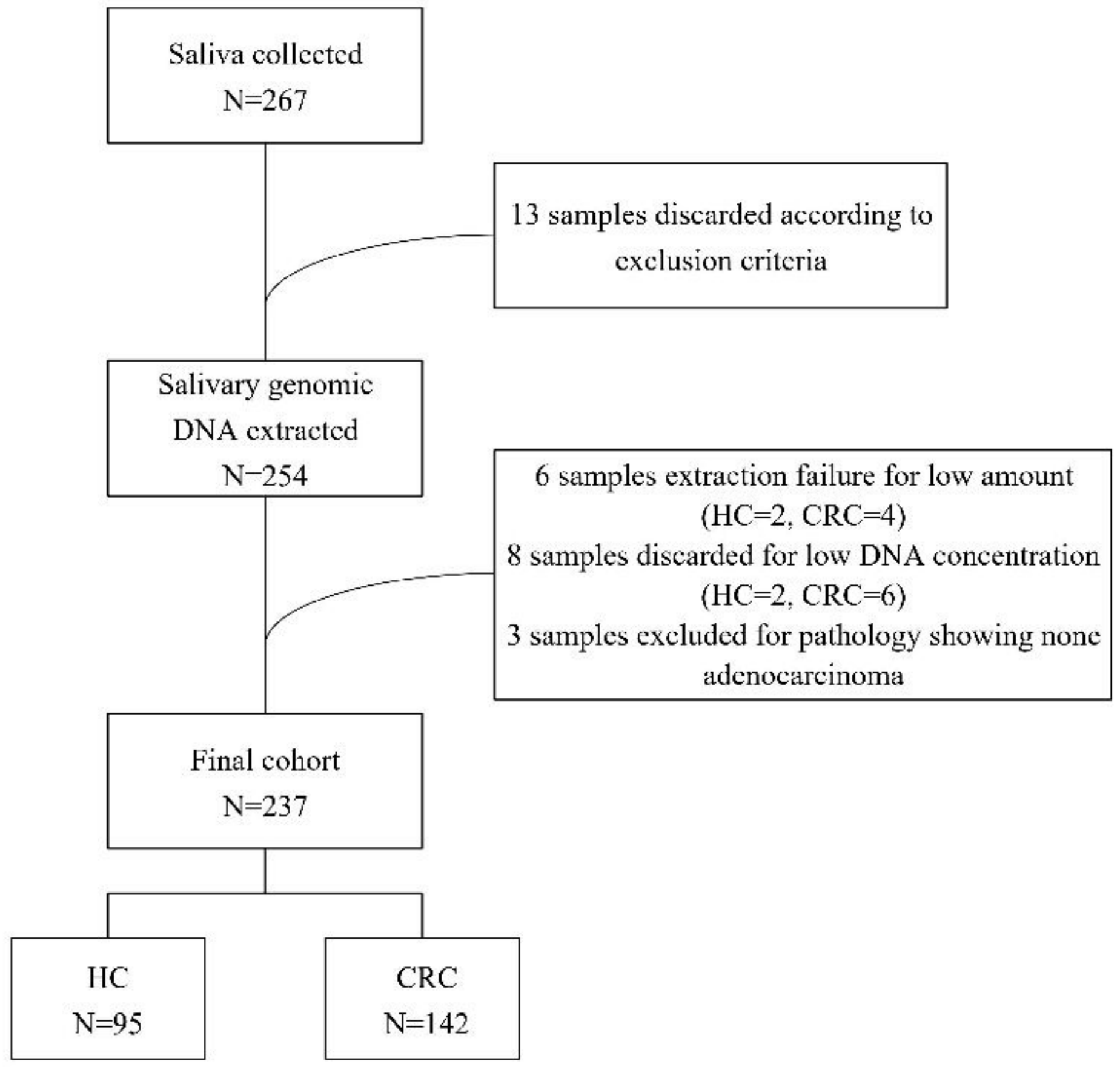

Figure 1

Workflow chart of the study. HC indicates healthy control; CRC, colorectal cancer. 
Saliva

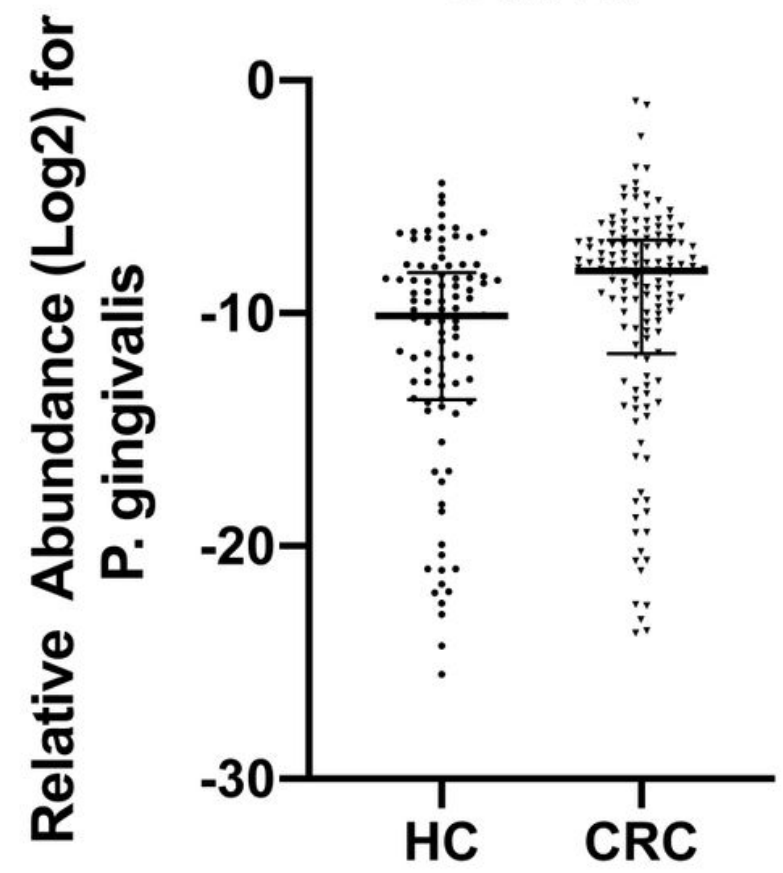

Saliva

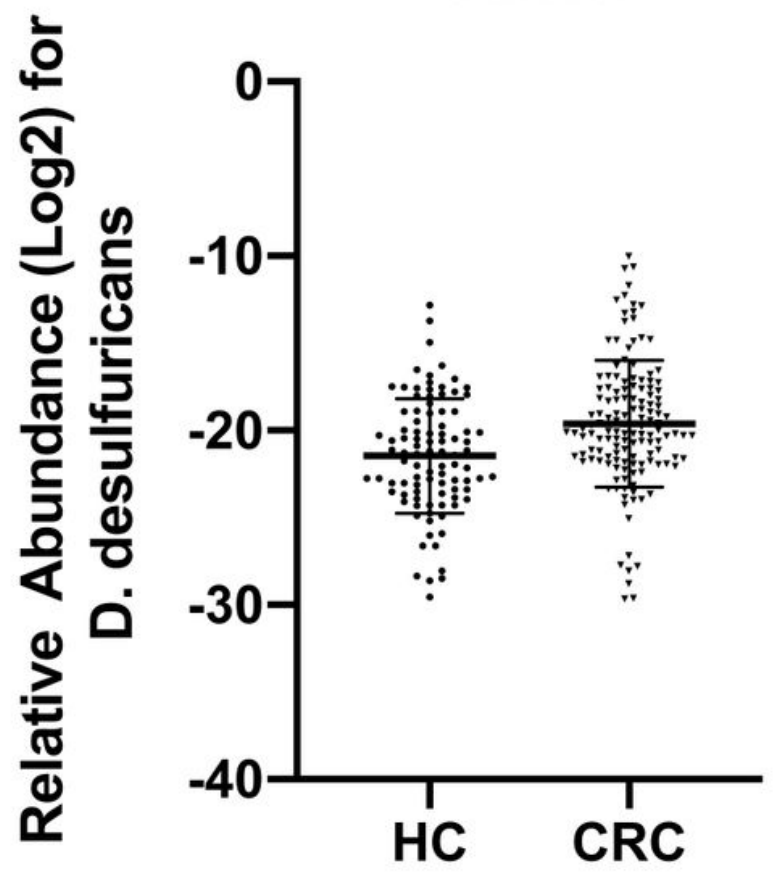

Figure 2

Scatter-plot diagram of relative salivary (left) Pg and (right) Dd level between HC and CRC group. The middle line indicates median or mean value while range between the upper and lower line indicates interquartile range or standard deviation, respectively. 
Points

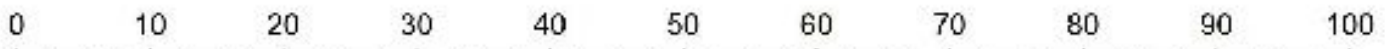

Age

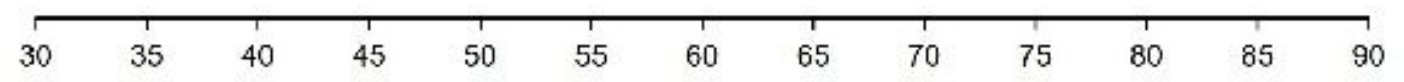

Sex

Female Male

$\mathrm{OHI}$

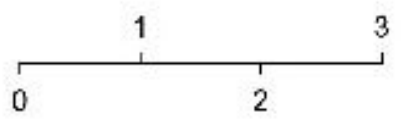

Dd

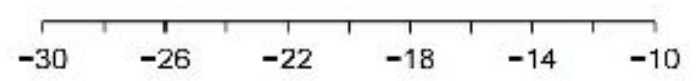

Total Points

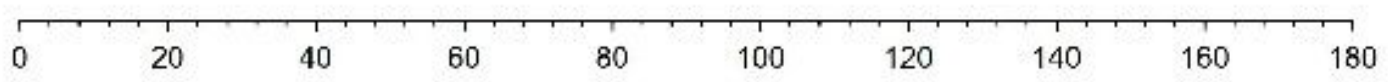

Probability of CRC

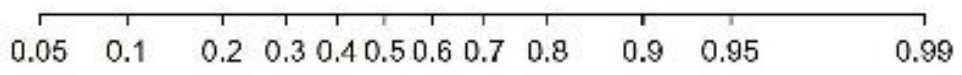

Figure 3

Nomogram for predicting the probability of CRC.
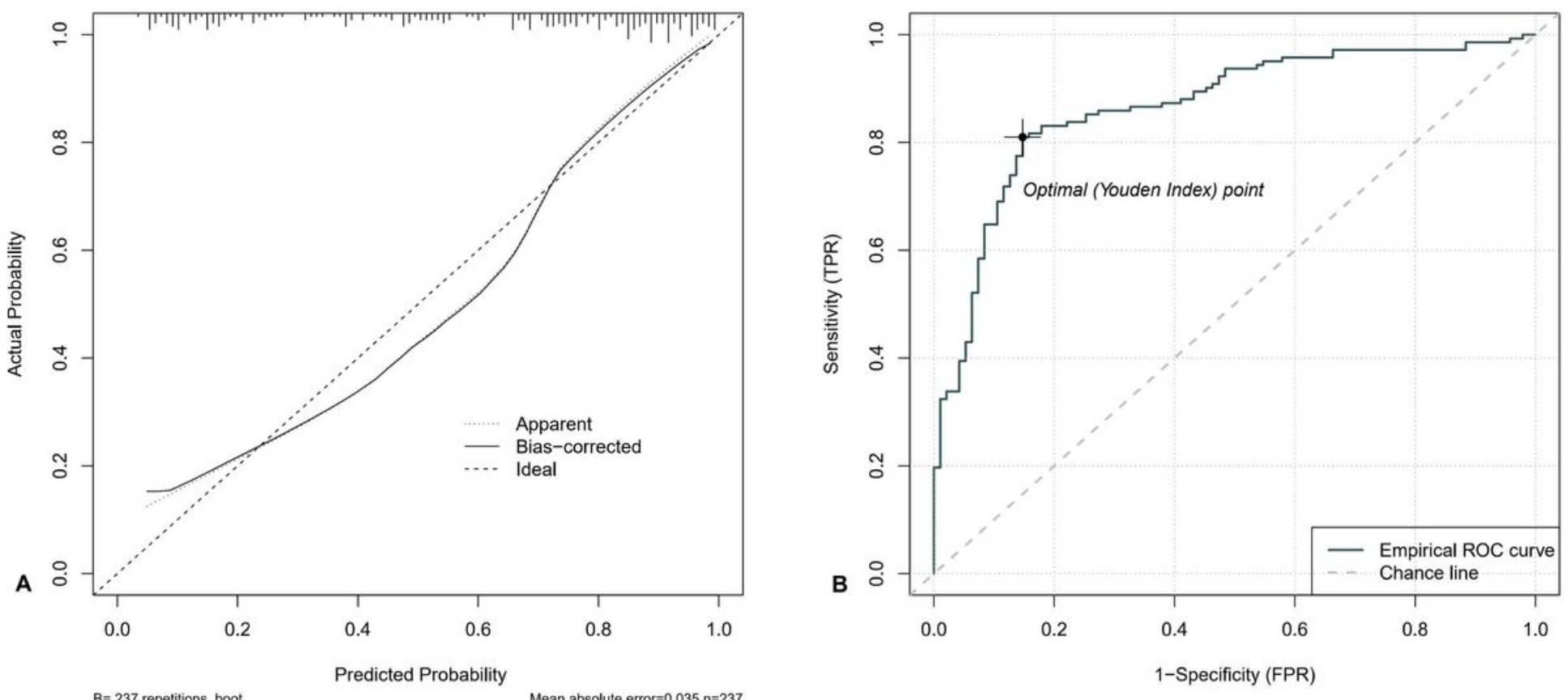

Figure 4 
Internal validation of the four-variable model. Calibration slope (left) of the four-variable model in the cohort. The $x$-axis indicates the predicted probability of CRC risk by the four-variable model while the $y$ axis is the actual value of the diagnosis. Calibration slope is 0.834 . Receiver operating characteristics (ROC) curve (right) for the four-variable model. C-index is 0.866 .

\section{Supplementary Files}

This is a list of supplementary files associated with this preprint. Click to download.

- Supplemmentarymaterials.docx

- Supplemmentarymaterials.docx

- Supplemmentarymaterials.docx 